\title{
Enhanced Mixing in Giant Impact Simulations with a New Lagrangian Method
}

Deng, Hongping ; Reinhardt, Christian ; Benitez, Federico ; Mayer, Lucio ; Stadel, Joachim ; Barr, Amy

\author{
$\mathrm{C}$
}

\begin{abstract}
Giant impacts (GIs) are common in the late stage of planet formation. The Smoothed Particle Hydrodynamics (SPH) method is widely used for simulating the outcome of such violent collisions, one prominent example being the formation of the Moon. However, a decade of numerical studies in various areas of computational astrophysics has shown that the standard formulation of SPH suffers from several shortcomings such as artificial surface tension and its tendency to promptly damp turbulent motions on scales much larger than the physical dissipation scale, both resulting in the suppression of mixing. In order to estimate how severe these limitations are when modeling GIs we carried out a comparison of simulations with identical initial conditions performed with the standard SPH as well as with the novel Lagrangian Meshless Finite Mass (MFM) method using the multimethod code, GIZMO. We confirm the lack of mixing between the impactor and target when SPH is employed, while MFM is capable of driving vigorous subsonic turbulence and leads to significant mixing between the two bodies. Modern SPH variants with artificial conductivity, a different formulation of the hydro force or reduced artificial viscosity, do not improve mixing as significantly. Angular momentum is conserved similarly well in both methods, but MFM does not suffer from spurious transport induced by artificial viscosity, resulting in a slightly higher angular momentum of the protolunar disk. Furthermore, SPH initial conditions unphysically smooth the core-mantle boundary, which is easily avoided in MFM.
\end{abstract}

DOI: https://doi.org/10.3847/1538-4357/aaf399

Posted at the Zurich Open Repository and Archive, University of Zurich

ZORA URL: https://doi.org/10.5167/uzh-182552

Journal Article

Published Version

Originally published at:

Deng, Hongping; Reinhardt, Christian; Benitez, Federico; Mayer, Lucio; Stadel, Joachim; Barr, Amy C (2019). Enhanced Mixing in Giant Impact Simulations with a New Lagrangian Method. The Astrophysical Journal, 870(2):127.

DOI: https://doi.org/10.3847/1538-4357/aaf399 


\title{
Enhanced Mixing in Giant Impact Simulations with a New Lagrangian Method
}

\author{
Hongping Deng ${ }^{1}\left(\mathbb{D}\right.$, Christian Reinhardt ${ }^{1}$, Federico Benitez ${ }^{1}$, Lucio Mayer ${ }^{1}$, Joachim Stadel $^{1}$, and Amy C. Barr ${ }^{2}$ (1) \\ ${ }^{1}$ Center for Theoretical Astrophysics and Cosmology, Institute for Computational Science, University of Zurich, Winterthurerstrasse 190, 8057 Zurich, Switzerland \\ hpdeng@physik.uzh.ch \\ ${ }^{2}$ Planetary Science Institute, 1700 E. Ft. Lowell, Suite 106, Tucson, AZ 85719, USA \\ Received 2018 March 30; revised 2018 November 9; accepted 2018 November 20; published 2019 January 16
}

\begin{abstract}
Giant impacts (GIs) are common in the late stage of planet formation. The Smoothed Particle Hydrodynamics (SPH) method is widely used for simulating the outcome of such violent collisions, one prominent example being the formation of the Moon. However, a decade of numerical studies in various areas of computational astrophysics has shown that the standard formulation of SPH suffers from several shortcomings such as artificial surface tension and its tendency to promptly damp turbulent motions on scales much larger than the physical dissipation scale, both resulting in the suppression of mixing. In order to estimate how severe these limitations are when modeling GIs we carried out a comparison of simulations with identical initial conditions performed with the standard SPH as well as with the novel Lagrangian Meshless Finite Mass (MFM) method using the multimethod code, GIZMO. We confirm the lack of mixing between the impactor and target when SPH is employed, while MFM is capable of driving vigorous subsonic turbulence and leads to significant mixing between the two bodies. Modern SPH variants with artificial conductivity, a different formulation of the hydro force or reduced artificial viscosity, do not improve mixing as significantly. Angular momentum is conserved similarly well in both methods, but MFM does not suffer from spurious transport induced by artificial viscosity, resulting in a slightly higher angular momentum of the protolunar disk. Furthermore, SPH initial conditions unphysically smooth the core-mantle boundary, which is easily avoided in MFM.
\end{abstract}

Key words: planets and satellites: formation - turbulence - methods: numerical

\section{Introduction}

During the late stage of terrestrial planet formation, energetic collisions between roughly Mars-sized planetary embryos are common (Chambers 2001). These collisions are called giant impacts (GIs) and influence the mass, spin, and the number of planets in the final planetary system. The outcomes of such violent collisions have been studied in many previous publications (Asphaug et al. 2006; Leinhardt \& Stewart 2012). One particularly compelling case is the GI hypothesis for the formation of the Moon (Cameron \& Ward 1976; Benz et al. 1986; Canup \& Asphaug 2001). The Moon and the Earth have almost identical isotope composition for several elements, such as oxygen (Wiechert et al. 2001) and titanium (Zhang et al. 2012). Either the impactor has very similar isotopic composition to the proto-Earth (Mastrobuono-Battisti et al. 2015; Dauphas 2017) or the impact mixes them efficiently assuming every planetary mass body has a unique isotopic signature (Kaib \& Cowan 2015) (see the review by Barr 2016). Many Smoothed Particle Hydrodynamics (SPH) simulations found that most disk silicates are derived from the impactor and mixing seems insufficient (Canup et al. 2013). Alternative models like a fast-spinning proto-Earth (Ćuk \& Stewart 2012), a hit and run collision (Reufer et al. 2012) and an impact between bodies of roughly equal mass (Canup 2012) have been proposed. However, all models are not entirely satisfactory because they either fail to explain the observations or introduce new issues, for example, forming a fast-spinning proto-Earth, which must be solved.

Most GI simulations have used SPH (Gingold \& Monaghan 1977; Lucy 1977). A few Eulerian code simulations are available, such as those using the FLASH code (Fryxell et al. 2000; Liu et al. 2015) and the CTH code (McGlaun et al. 1990; Canup et al. 2013). Many shortcomings of SPH have been exposed and overcome in the past few years, such as the artificial tension force acting at the interface between two fluids (Agertz et al. 2007; Price 2008), and the excessive numerical viscosity in shear flow (Cullen \& Dehnen 2010). A new SPH formulation has been proposed (Hopkins 2013; Saitoh \& Makino 2013) and used in GI simulations by Hosono et al. (2016). Special techniques for SPH are also developed in GI simulations, such as the treatment of free surface and the explicit conservation of entropy (Reinhardt \& Stadel 2017). Discreteness particle noise in SPH and artificial viscosity smear out local velocity variations thus damping subsonic turbulence on overly large scales relative to the physical dissipation scales of the turbulent cascade (Bauer \& Springel 2012). These issues have promoted improvements of the method (Beck et al. 2016) that are absent in all previous GI simulations using SPH. Alternatively, other hydrodynamical solvers have recently been developed that still keep the main advantage of SPH in treating collisions between bodies, namely its Lagrangian nature. Hopkins (2015) implemented a new Lagrangian meshless finite mass (MFM) method in the GIZMO code showing excellent shock capturing and conservation properties (Hopkins 2015; Deng et al. 2017). Hopkins (2015) also shows that MFM can capture small-scale turbulence, yielding results that are very similar to those of moving-mesh and stationary-grid methods. GIZMO MFM also appears to sustain subsonic MRI (Balbus \& Hawley 1991) turbulence much longer than SPH in local shearing box simulations (H. Deng et al. 2019, in preparation).

We ran GI simulations using the multimethod GIZMO code (Hopkins 2015), employing both MFM and SPH for different equations of state and planetary compositions to investigate the role of the numerical hydrodynamics method on mixing in the post-impact target. We also analyzed the protolunar disk's 
dynamic property and composition. The main features of the hydrodynamical methods adopted and the initial conditions of GIs are described in Section 2. We present the results of single component impacts in Section 3.1 as well as multiplecomponent impacts in Section 3.2. We discuss the results in Section 4 and draw conclusions in Section 5.

\section{Numeric Methods}

\subsection{The Hydro-methods}

We use the GIZMO code (Hopkins 2015), which includes a number of particle-based hydro solvers, and have augmented them with new equations of state in order to be able to model GIs. In particular, we use the standard SPH solver inherited from the GADGET3 code (see Springel 2005), which is based on the density-energy formulation of the SPH equations and adopts standard Monaghan artificial viscosity with the Balsara switch (Balsara 1995) to minimize viscous dissipation away from shocks. The other numerical hydrodynamics method that we consider is MFM, which solves the hydro equations by partitioning the domain using volume elements associated with the original particle distribution, and computing fluxes at the interfaces of the resulting tessellation by means of a Riemann solver as in finite volume Godunov-type methods (Hopkins 2015). While many modern SPH variants have appeared in the last years that improve considerably in its ability to model complex flows, we chose to use this relatively old SPH formulation to enable comparison with most past work. However, we tested the effect of improvements present in modern SPH codes such as the Cullen \& Dehnen artificial viscosity switch (Cullen \& Dehnen 2010) and the artificial thermal conductivity of Read \& Hayfield (2012) in the discussion section (see Section 4). Hosono et al. (2016) presented GI simulations with density independent SPH (DISPH; Hopkins 2013; Saitoh \& Makino 2013). However, it is not trivial to enable a nonideal equation of state (EOS) in DISPH (Hosono et al. 2013). We present no DISPH simulations since DISPH also damps subsonic turbulence (our focus of the paper, see Figures 4, 5) as SPH (Hopkins 2015).

The newest version of GIZMO (Hopkins 2017) supports a general EOS (including Tillotson EOS interface) implemented by the author of the code. We added in our own EOS interface. The HLLC (Harten-Lax-van Leer-Contact) Riemann solver (Toro et al. 1994) is extended for general EOS by doing explicit state reconstruction for the sound speed and internal energy. The Riemann solver works well with general EOS, see Appendix A. In order to assess numerical issues due to this generalized Riemann solver we also tested a more accurate contact wave estimation proposed by $\mathrm{Hu}$ et al. (2009) but find that there is no noticeable difference to the default HLLC solver so we did not use it in the simulations presented in this paper.

We use the Tillotson EOS (Tillotson 1962) to model impacts of undifferentiated objects and ANEOS/M-ANEOS (Thompson \& Lauson 1974; Melosh 2007) for a multiple-component impact model (a differentiated structure with $30 \%$ iron-ANEOS-and $70 \%$ dunite-M-ANEOS - by mass). The Tillotson EOS does not yield a thermodynamically consistent treatment of mixtures between two phases, and cannot model the critical behavior at phase transitions (Brundage 2013). However, pressure-release melting might happen when the highly compressed core is unloaded from equilibrium (Asphaug et al. 2006). We show that the ANEOS EOS does capture the phase transition in the iron core when it is strongly disturbed in Appendix B. We use 1 Earth radius $\left(R_{\oplus}\right), 1 \mathrm{~km} \mathrm{~s}^{-1}$ with the gravitational constant equal to 1 as our unit system. We describe our core-mantle boundary treatment in the following section.

\subsection{Initial Conditions and the Core-mantle Boundary}

We follow Reinhardt \& Stadel (2017) to produce a low noise representation of a planet's equilibrium initial conditions based on equal area tessellations of the sphere. The initial setups are further relaxed by running them with the hydro code chosen for the run (standard SPH or MFM) for about $3 \mathrm{hr}$ of simulation time until the random velocity of particles, measured by their root-mean-square velocity, is less than $1 \%$ of the impact velocity. In order to avoid problems at the planet's surface while relaxing the model, we applied the free surface treatment proposed in Reinhardt \& Stadel (2017) but disabled it during the impact simulation to allow for a direct comparison with published results. Removing the free surface treatment has no effects on the planets thermal state on the short timescale of the initial collision, except in the very outer part.

We use $500 \mathrm{~K}$ particles (comparable to recent highresolution impact simulations) to sample the target $\left(0.89 M_{\oplus}\right)$ in the canonical Moon formation scenario (Canup et al. 2013). It is isentropic with an entropy of $1200 \mathrm{~J} \mathrm{~kg}^{-1} \mathrm{~K}^{-1}$ in the core and $2700 \mathrm{~J} \mathrm{~kg}^{-1} \mathrm{~K}^{-1}$ in the mantle (see Barr 2016, for details). In SPH, the density of the $i$ th particle is the kernel weighted sum of its neighbor particles' masses (Springel 2005);

$$
\rho_{i}=\Sigma_{j} m_{j} W\left(\left|\boldsymbol{r}_{i}-\boldsymbol{r}_{j}\right|, h_{i}\right)
$$

as a result, the core-mantle boundary is not infinitely sharp. The core-mantle transition is at the smoothing length scale in SPH while MFM has a larger transition region (see the upper panel of Figure 1). Particles/cells in the transition region with a density intermediate between that of iron and dunite do not have well defined physical properties. They are expanded iron or compressed dunite in the EOS table, which is not physically motivated.

Additionally, at the core-mantle boundary, the density, and thus the smoothing length, changes sharply. This leads to an artificial tension force separating the two components in standard SPH (Agertz et al. 2007; Price 2008). In the lower panel of Figure 1, for the SPH realization, we notice a discontinuous pressure profile when employing the M-ANEOS EOS. This is caused by artificial surface tension. Instead, MFM delivers a continuous pressure profile, albeit still exhibiting a small pressure bump. Surface tension prevents fluid mixing (Agertz et al. 2007), but preserves a sharper core-mantle boundary in standard SPH compared to MFM (see Figure 1).

Woolfson (2007) proposed an extra correction factor for the density at the interface between different components to maintain a sharp core-mantle transition. However, this is an ad hoc correction that is not formally consistent with the SPH or MFM formulation. We follow a different strategy and use particles with different masses in our MFM model. We recall that, in MFM, the density of the $i$ th particle is:

$$
\rho_{i}=\frac{m_{i}}{V_{\mathrm{eff}, i}}
$$

where $V_{\mathrm{eff}, i}$ is the effective volume of the $i$ th particle (see Hopkins 2015). Using iron particles of mass two times that of the dunite particles', the smoothing length, thus $V_{\text {eff }}$, is almost continuous across the core-mantle boundary, yet we obtain a sharp core-mantle boundary with no particles entering an 

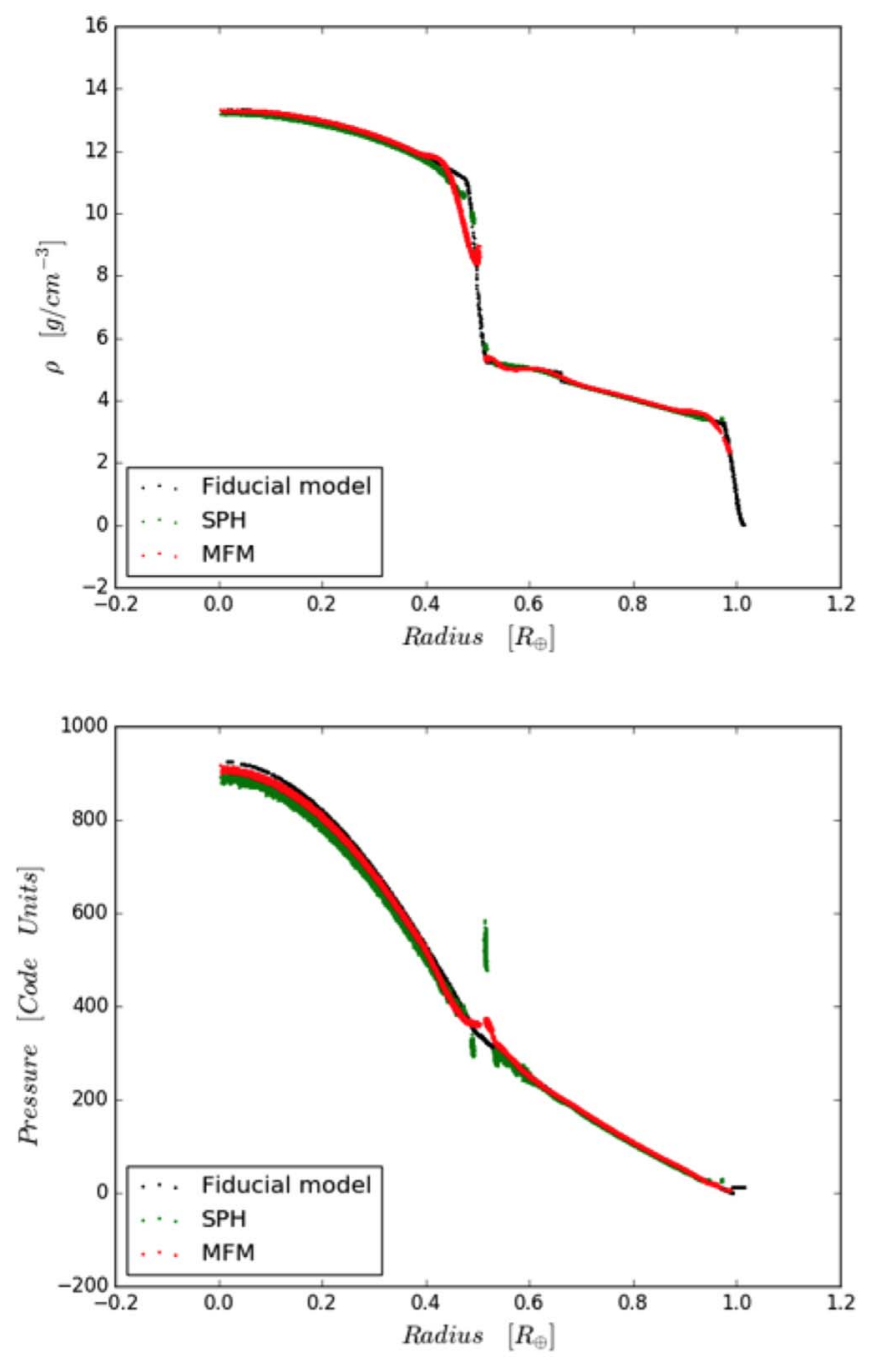

Figure 1. Density (upper panel) and pressure (lower panel) profile of the $0.89 M_{\oplus}$ target in the benchmark moon formation run119 of Barr (2016). The initial condition is modeled with $500 \mathrm{~K}$ particles of equal mass. The CTH grid code model (fiducial model), SPH model, and MFM model are shown in black, green, and red respectively. Some particles/cells enter an unphysical state in the core-mantle transition region in all three models with the SPH model showing a noncontinuous pressure profile at the core-mantle boundary.

unphysical state (see upper panel of Figure 2). Woolfson (2007) had to vary the correction factor according to the density ratio of the two components whereas with our approach we simply use a 2:1 mass ratio of particles. Indeed moderate variations in the density ratio are tolerable while a timedependent variation of particle mass would cause the method to fail. In Figure 2, the pressure is still continuous in the MFM model, and overlaps with the fiducial model, while the SPH model still suffers from artificial tension force and has particles entering unphysical states. In the impact simulations, we use different mass particles in MFM but the same mass particles for SPH to enable direct comparison with prior work. An alternative SPH formulation (Ott \& Schnetter 2003) based on discretizing the particle number density instead of mass density, similarly to the density estimate approach in MFM, can also resolve the sharp core-mantle boundary. Recent tests using a similar scheme (Solenthaler \& Pajarola 2008) found that it is difficult to build equilibrium models of planetary bodies. As a
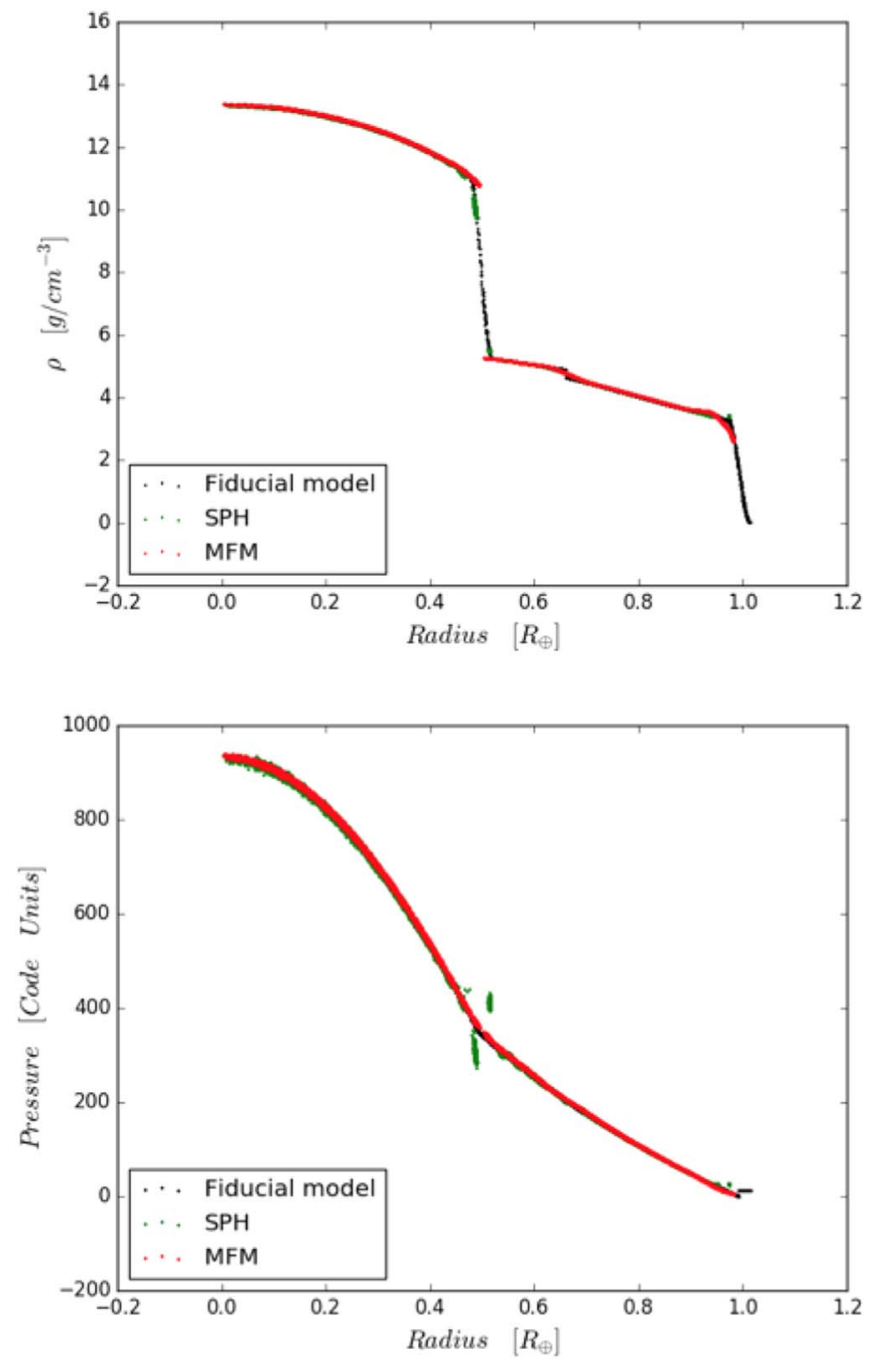

Figure 2. Density (upper panel) and pressure (lower panel) profiles of the $0.89 M_{\oplus}$ target in the benchmark Moon formation run119 of Barr (2016). The initial condition is modeled using $500 \mathrm{~K}$ particles with iron particles' mass equal two times dunite particles' mass. The CTH grid code model (fiducial model), SPH model and MFM model are shown in black, green and red respectively. Only the MFM model keeps the infinitely sharp (no low-density iron particles) core-mantle transition, while the SPH model still has a noncontinuous pressure profile at the core-mantle boundary.

result this method might not be suitable for planetary-size collisions (A. Emsenhuber 2018, private communication).

We note that we use different mass particles for iron and dunite but that these masses are the same in both the impactor and target. Using different iron/dunite particle masses in the impactor and target can lead to numerical differentiation and thus cause unphysical mixing in our test runs with MFM.

\section{Results}

\subsection{Single Component Impact}

For the single component models, we use the Tillotson EOS because it is simple and highly reliable. This EOS can accurately model shocks, which are very important in highspeed impacts, and shows good agreement to measured data (Brundage 2013). Its main weakness is that it does not provide a thermodynamically consistent treatment of vaporization, which is not an issue in this simulation as we mainly focus on the different inner structure of the post-impact target here. 

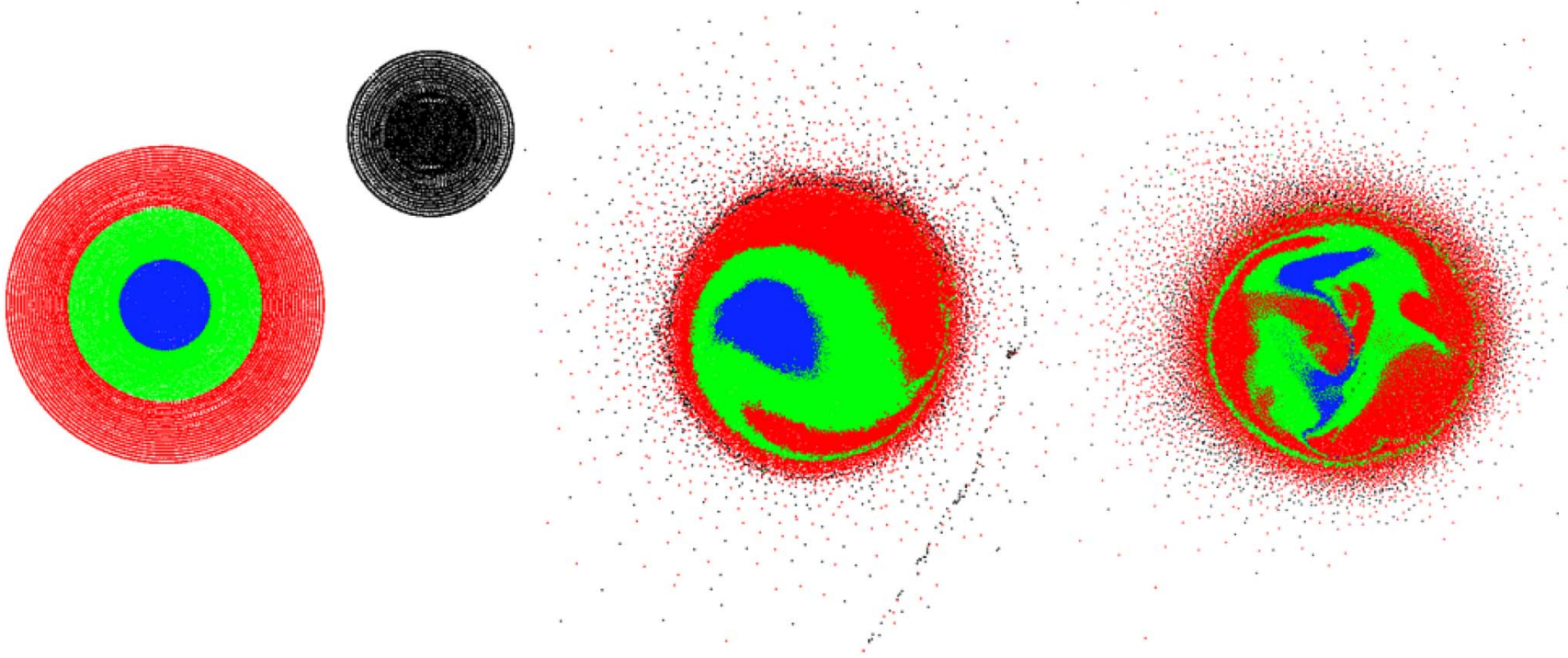

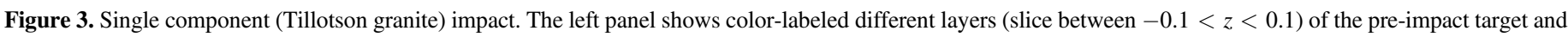

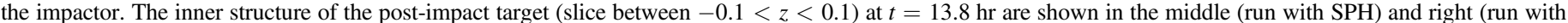

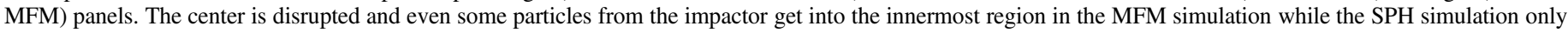
show moderate deformation of the target.

We use $500 \mathrm{~K}$ particles to represent a $1 M_{\oplus}$ target and a $0.1 M_{\oplus}$ impactor, both of which are composed of granite described by the Tillotson EOS. This one component model is free of core-mantle discontinuity, which is hard to handle in numeric models, see the discussion in Section 2.2. The impact setup is similar to the canonical Moon formation impact of Canup et al. (2013). The impact velocity equals $10 \mathrm{~km} \mathrm{~s}^{-1}(1.1$ times of their mutual escape velocity) and the impact parameter $b=0.71$ corresponds to an impact angle of $45^{\circ}$. The initial separation between the two bodies is $0.4 R_{\oplus}$. We run this simple impact with both standard SPH and MFM implementations in the GIZMO code, hence the only difference is the hydro-method.

We observe a striking difference in the inner structure of the post-impact target between MFM and standard SPH. In Figure 3, we mark three layers of the pre-impact target and the impactor with four different colors to trace the deformation of the target and the spread of the impactor. In the SPH simulation, the target's center deforms slightly while in the MFM simulation the center is dispersed throughout the body. In the SPH simulation, the outermost layer is strongly deformed but never penetrates the central region. The MFM method, instead, allows fluid elements from the outermost layer of the target to mix into the innermost region.

This mixing happens as a result of complex 3D subsonic turbulence whose characteristic velocity amplitude is less than $1 \mathrm{~km} \mathrm{~s}^{-1}$. Figure 4 shows the velocity field around the $z=0$ plane after the GI. In the SPH run, the flow is almost laminar and simply circulates around a low-velocity center. The flow structure is influenced by the tidal force from the ejecta and by their fallback. In the MFM run, we always observe significantly more substructure in the flow characterizing the post-impact target. Our findings echo the analysis carried out by Bauer \& Springel (2012), who showed that standard SPH results in a dissipation scale for turbulence that is unphysical and much higher than that of finite volume methods using static or moving meshes. We expect behavior of MFM in this domain to be closer to the latter codes than to SPH as a result of the

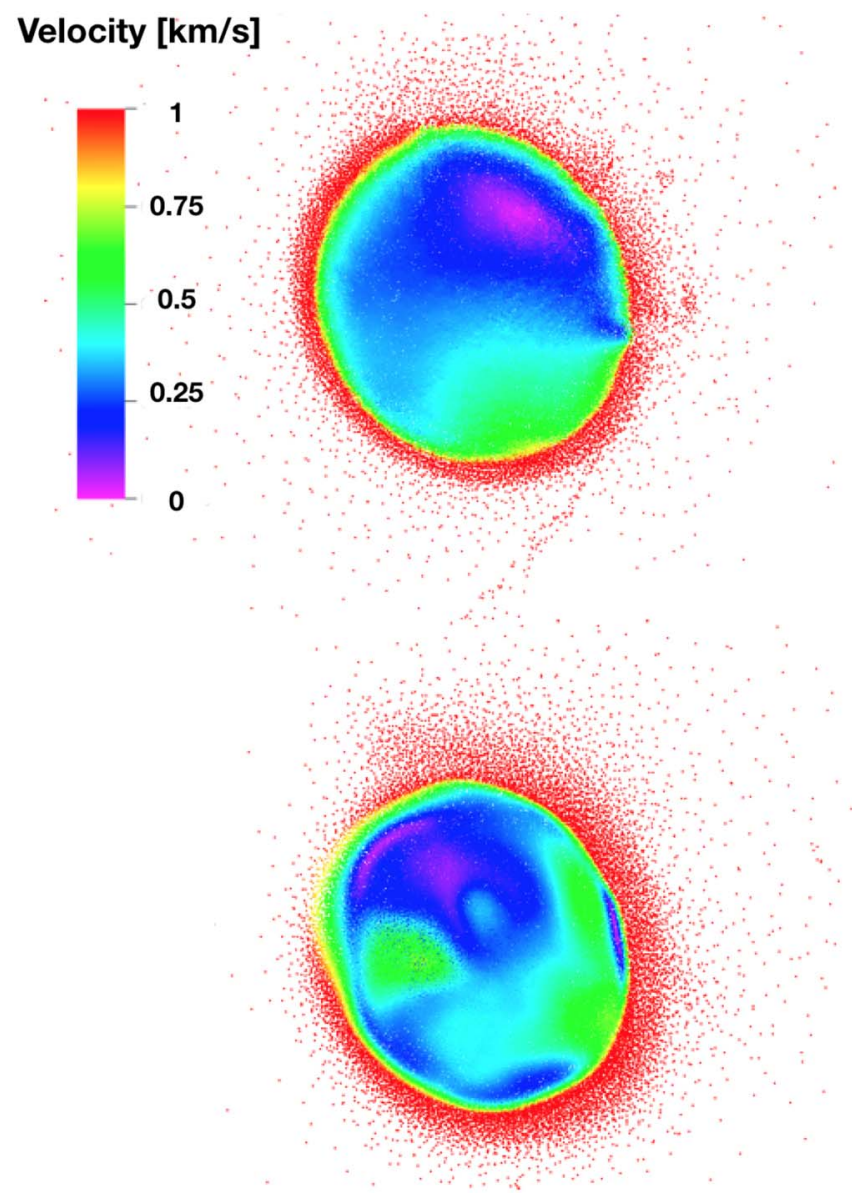

Figure 4. Velocity magnitude of the $-0.1<z<0.1$ region in the major body of the single component impact Section 3.1. The snapshots are taken at $t=10.5 \mathrm{hr}$ and some clumps are still recolliding with the major body. The upper panel is the SPH simulation and the lower panel is the MFM simulation. MFM is able to capture the more complex subsonic turbulence while SPH tends to damp it readily on large scales, resulting in a more coherent flow rotating around a low-velocity center. 
Table 1

Comparison Between SPH and MFM Simulations

\begin{tabular}{lccccccc}
\hline \hline Run & $b$ & $\frac{v_{\text {imp }}}{v_{\text {esc }}}$ & $\frac{L_{D}}{L_{\mathrm{EM}}}$ & $\frac{M_{D}}{M_{L}}$ & $F_{D, \operatorname{tar}}$ & $\frac{M_{\mathrm{Fe}}}{M_{D}}$ & $\delta f_{T}$ \\
\hline 1 & 0.72 & 1.0 & 0.35 & 1.70 & 0.27 & 0.07 & -0.70 \\
2 & 0.64 & 1.0 & 0.05 & 0.28 & 0.47 & 0.10 & -0.48 \\
3 & 0.64 & 1.1 & 0.12 & 0.63 & 0.45 & 0.16 & -0.50 \\
4 & 0.72 & 1.0 & 0.35 & 1.72 & 0.30 & 0.07 & -0.67 \\
\hline 5 & 0.72 & 1.0 & 0.37 & 1.86 & 0.43 & 0.04 & -0.50 \\
6 & 0.64 & 1.0 & 0.06 & 0.43 & 0.82 & 0.04 & -0.08 \\
7 & 0.64 & 1.1 & 0.12 & 0.71 & 0.62 & 0.15 & -0.30 \\
\hline
\end{tabular}

Note. Runs 1-4 are SPH simulations while runs 5-7 are MFM simulations. Runs 1-3 use equal mass rock/iron particles as in most previous studies while runs 4-7 use rock/iron particles of 1:2 mass ratio (see the discussion in Section 2.2).

absence of explicit numerical dissipation from artificial viscosity and because of higher accuracy of velocity variations computed by means of the Riemann solver. It is aligned with the outcome of the many numerical tests discussed in Hopkins (2015). In the following section, we will assess the importance of capturing mixing promoted by (subsonic) turbulence in the context of the canonical Moon-forming impact.

\subsection{Multiple-component Impact}

We simulated "run119" described by Canup et al. (2013) with SPH and MFM using $500 \mathrm{~K}$ particles. In this impact, a $0.89 M_{\oplus}$ target is hit by a $0.13 M_{\oplus}$ impactor at their mutual escape velocity $\sim 9 \mathrm{~km} \mathrm{~s}^{-1}$. This model was proposed as a benchmark by Barr (2016; see Section 2.1). M-ANEOS coefficients and other details about the simulation setup may be found there. We note that we choose this model just because the Moon-forming impact is well studied. We are not trying to solve the isotope conundrum in the GI hypothesis of the Moon formation (Asphaug 2014) here but focusing on the different mixing in general impacts caused by the hydro-method. We also vary the impact velocity and angle and summarize our simulations in Table 1.

\subsubsection{Protolunar Disk Property}

We carry out the analysis of the protolunar disk following Canup et al. (2013). We calculate the disk mass $M_{D}$ and disk angular momentum $L_{D}$ at $t=35 \mathrm{hr}$, when the properties of the disk no longer change significantly. In our SPH simulation (run1), we get a disk mass $M_{D}=1.70 M_{L}$ and disk angular momentum $L_{D}=0.35 L_{\mathrm{EM}}$, which is close to the results of the highest resolution simulation for run $119, M_{D}=1.69 M_{L}$ and $L_{D}=0.33 L_{\mathrm{EM}}$ (Canup et al. 2013). Here, $M_{L}$ and $L_{\mathrm{EM}}$ are, respectively, the Moon mass and the angular momentum of the Earth-Moon system. Our SPH simulation agrees very well with previous SPH simulations. By comparing run4 with run1, which uses particles with different masses in SPH, we conclude that changing the mass of particles does not make a significant difference. In our MFM simulation (run5), we have $M_{D}=1.86 M_{L}, L_{D}=0.37 L_{\mathrm{EM}}$. Comparing runs $5-7$ to $1-3$, MFM simulations have larger disk mass and angular momentum than their SPH counterparts, which we attribute to more accurate handling of angular momentum transport in MFM for differentially rotating flows (Deng et al. 2017) We note that, while SPH conserves angular momentum by construction, the inclusion of artificial viscosity causes dissipation that enhances angular momentum transport.

\subsection{Mixing}

In the canonical Moon formation scenario, the portion of the impactor that avoids colliding with the proto-Earth is sheared into spiral ejecta. The ejecta will contract and recollide with the target and lead to the tidal disruption of the former and the formation of the disk. In this model, most of the disk matter comes from the tidal disruption of the impactor. In run119 of Canup et al. (2013), 70\% of the disk material originates from the impactor.

Following Reufer et al. (2012) we use the deviation factor $\delta f_{T}$ to characterize the mixing in the Moon-forming GI, where

$$
\begin{gathered}
f_{T}=\left(M_{\mathrm{targ}}^{\text {silc }} / M_{\mathrm{tot}}^{\text {silc }}\right)_{\mathrm{disk}}, \\
\delta f_{T}=\frac{\left(M_{\mathrm{targ}}^{\text {silc }} / M_{\mathrm{tot}}^{\text {silc }}\right)_{\mathrm{disk}}}{\left(M_{\mathrm{targ}}^{\text {silc }} / M_{\mathrm{tot}}^{\text {silc }}\right)_{\text {post-impactTarget }}}-1 .
\end{gathered}
$$

$M_{\mathrm{targ}}^{\text {slic }}$ and $M_{\mathrm{tot}}^{\text {slic }}$ denote the mass of the silicate part of the disk/ post-impact target derived from the target and the total disk/ post-impact target mass, respectively. $\delta f_{T}$ measures the composition similarity between the silicate part of the protolunar disk and the post-impact target. In our SPH simulation, $f_{T}=27 \%, \delta f_{T}=-70 \%$ agrees well with $f_{T} \approx 30 \%$ in Reufer et al. (2012) and Canup et al. (2013). In the MFM simulation, $f_{T}=43 \%, \delta f_{T}=-50 \%$ and there is a higher degree of mixing. This trend holds when we vary the impact angle and velocity (see Table 1).

Similar to the single component model (Figure 4), MFM captures more complex turbulence if multiple components impact (see Figure 5). In Figure 6 we can clearly appreciate how different the mixing in the two methods is. We label with different colors the two layers of the proto-Earth mantle and core and the impactor's mantle and core to trace the components. In the SPH simulation, the two layers of the mantle are distorted and become intertwined but do not mix (see the snapshot taken at $t=36 \mathrm{hr}$ ). However, MFM mixes the two layers of the proto-Earth mantle and the impactor mantle thoroughly and quickly (snapshot taken at $t=14 \mathrm{hr}$ ).

The extent of mixing (see Figure 6) in the multicomponent Moon formation simulation is much more pronounced than in the single component model in Figure 3. The iron core can reflect pressure waves and shorten the sound crossing timescale in the post-impact target. This facilitates mixing in the postimpact target. The tidal interaction between the core and mantle also drives turbulence and enhances mixing. In the SPH simulations, silicates from the impactor always stay on the surface of the post-impact target. They originate from fallback ejecta. The artificial surface tension (see Section 2.2) prevents them from entering the inner part of the post-impact target (Hosono et al. 2016), while the suppression of turbulence in the post-impact target (see Figure 5) prevents them from mixing with the target further. These two numerical effects in SPH tend to increase the concentration of the impactor material at the surface layer of the target. Some fallback clumps are able to accelerate fluid elements across the surface layer of the target, and then launch them onto disk-like orbits. As a consequence, in the SPH simulation more impactor material, 

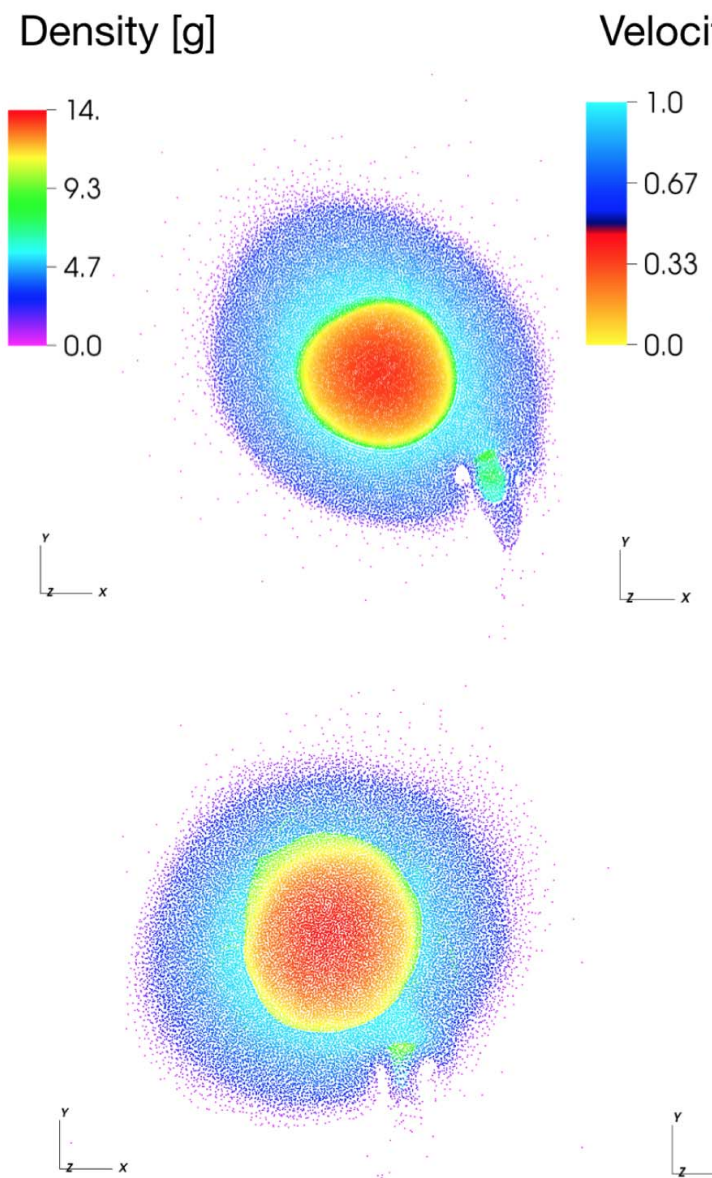

Velocity $[\mathrm{km} / \mathrm{s}]$
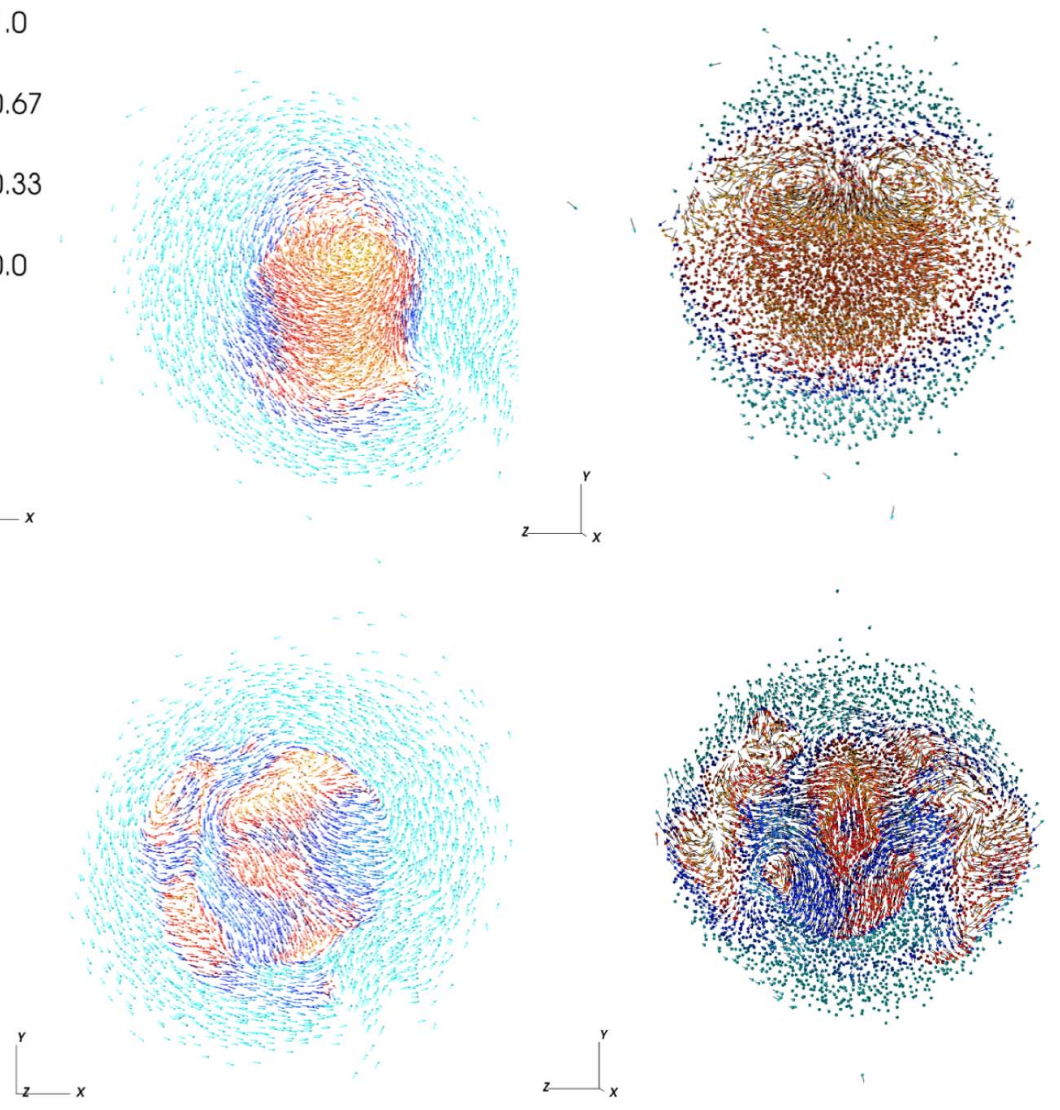

Figure 5. Snapshots of multiple-component impact run 3 (SPH, three upper panels) and run 7 (MFM, three lower panels) at $\sim 7$ hr. Left two panels: density plot (slice between $-0.05<z<0.05$ ) of the post-impact target. SPH artificial tension force causes numeric particle separation, which is absent in the MFM simulation. The other four panels show the flow structure in the post-impact target in the $x-y$ plane (middle, impact plane) and the $y-z$ plane (right). MFM captures much more complex three-dimensional subsonic turbulence than $\mathrm{SPH}$, which is crucial to follow the mixing during the whole simulation time.
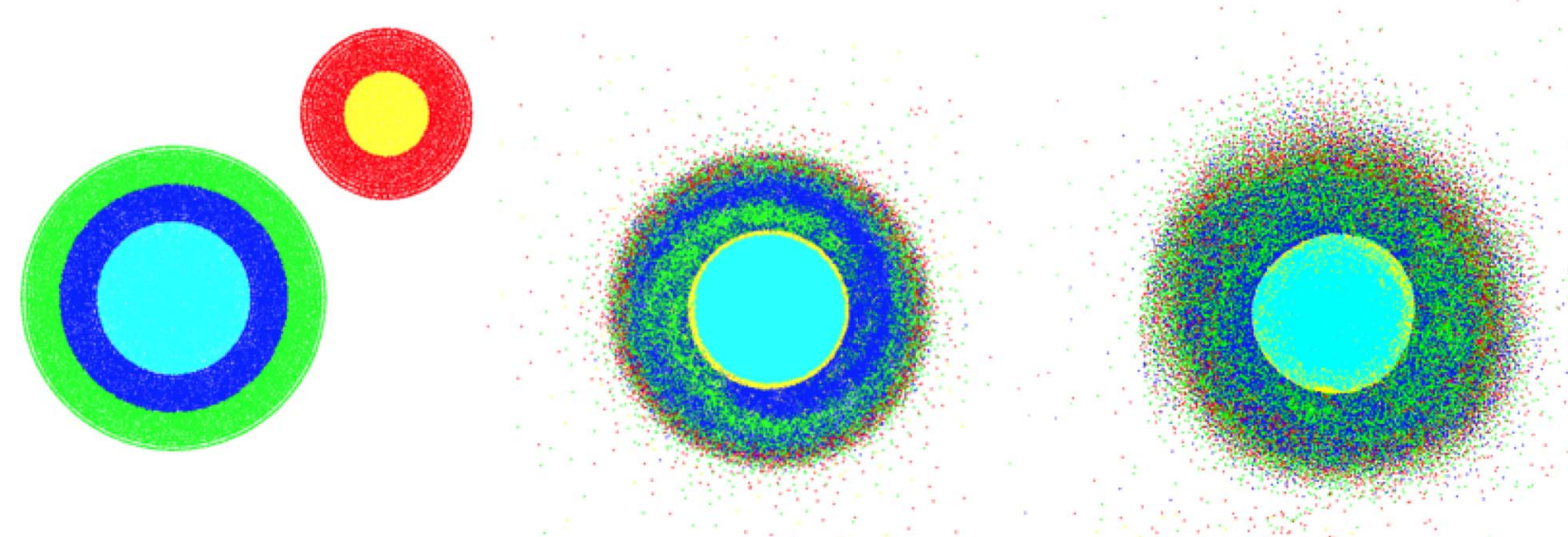

Figure 6. Multiple-component impact with ANEOS/M-ANEOS. Left panel: color-labeled different layers (slice between $-0.1<z<0.1$ ) of the pre-impact target (core and two layers of mantle) and the impactor (core and mantle). Middle panel: the material distribution at $t=36 \mathrm{hr}$ in the SPH run. Right panel: the material distribution at $t=14 \mathrm{hr}$ in the MFM run. In the SPH simulation, particles from the impactor mantle stay on the surface of the post-impact target due to the artificial tension force at the surface of the target and suppression of turbulence in the inner part of the target, which is also shown in Emsenhuber et al. (2017). However, MFM mixes the post-impact target thoroughly and quickly. MFM has a puffy planet surface, which is similar to the density independent SPH of Hosono et al. (2016).

which should have mixed deeper into the target, can be ejected. On the other hand, MFM mixes the impactor's mantle and the target quickly, hence more silicates from the target can be propelled into the protolunar disk.

\section{Discussion: Variants of the SPH Method}

In the previous sections, we have shown how MFM can resolve subsonic turbulence and the associated mixing in GIs, 
which standard SPH cannot. The artificial tension force of standard SPH prevents fluid mixing, which in turn prevents fallback ejecta from mixing with the post-impact target (Section 3.3). Artifacts due to artificial surface tension can be alleviated in SPH by introducing a conductivity term in the hydro equations (Price 2008; Read \& Hayfield 2012), or by employing a more accurate integral-based gradient estimator (Garcia-Senz et al. 2012; Rosswog 2015). We tested the former improvement. We reran the SPH simulation of run119 with artificial conductivity as suggested by Read \& Hayfield (2012). Mixing in the post-impact target is marginally improved, with the impactor's mantle penetrating a little deeper and the two layers of the target's mantle fracturing after a strong distortion rather than remaining intact as in standard SPH (see Figure 6). However, this run also results in iron particles floating on the post-impact target's surface, which is likely caused by the complex EOS (see Appendix A of Saitoh \& Makino 2016).

Concerning other improvements that we did not test, it should be recalled that, since mixing is aided by the development of subsonic turbulence triggered by the collision, the ability to capture the latter phenomenon should be considered as a requirement for any SPH variant to be capable of modeling the correct physical behavior in GIs. This is additional to removing artificial surface tension. In this respect Hopkins (2015) showed that DISPH does not help to sustain subsonic turbulence, although Wadsley et al. (2017) found considerable benefits when a similar approach is combined with higher order kernels and a turbulent diffusion term. Beck et al. (2016) shows their improved Cullen \& Dehen switch helps to sustain subsonic turbulence. We also rerun the same simulation with the Cullen \& Dehnen artificial viscosity prescription but did not find any noticeable difference in the mixing. In summary, so far we could not determine if there is any combination of the many proposed improvements to standard SPH that can capture turbulence and mixing in the context of GIs, which MFM can do by design.

\section{Conclusions and Perspectives}

We employed both SPH and, for the first time, a new Lagrangian method (MFM) to carry out GI simulations. Our goal was to compare their outcomes and determine if the degree of mixing depends on the numerical technique. In our single component model with the Tillotson EOS, we find that turbulence, and thus mixing, is suppressed in the SPH simulation. We then simulated the canonical Moon formation model with the M-ANEOS EOS. Our MFM initial conditions accurately model the core-mantle boundary with no particles entering an unphysical state. Our SPH results are consistent with previous results reported in the literature. The MFM simulations agree well with SPH simulations in terms of disk mass and angular momentum but show a marked increase in the mixing between the impactor and the target.

MFM is a well-established hydrodynamics method with no numerical features that would exaggerate the mixing seen in these simulations. Instead, the implication from our work is that previous simulations have underestimated the amount of mixing that happens in real impacts, which is in-line with notorious problems of standard SPH in capturing mixing in other astrophysical applications (Agertz et al. 2007; Wadsley et al. 2017). However, the outcomes for the canonical Moonforming impact obtained here still have disks originating primarily from the impactor. Fully resolving the isotope conundrum arising in the Moon formation GI theory (Asphaug 2014) likely requires different initial conditions for the encounter. Hit-and-run models, for example, those in Reufer et al. (2012), could potentially result in a more efficient mixing, provided enough material is launched into orbit to create a satellite of lunar mass. Based on our results, MFM would seem to be an ideal method to pursue further studies of mixing under a variety of initial conditions of GIs. This work simply represents the first step in this direction.

We thank Stephan Rosswog, Philip Hopkins, Romain Teyssier, and Martin Jutzi for useful discussions and James Wadsley for careful and useful comments on the first version of this paper that helped to improve it considerably. We thank the referee, Alexandre Emsenhuber, and the other anonymous referee for suggestions that improved the paper. We acknowledge support from the Swiss National Science Foundation via the National Center for Competence in Research (NCCR) PlanetS. C.R. acknowledges support from SNF Grant in "Computational Astrophysics" (200020 162930/1). Author Barr acknowledges support from NASA Emerging Worlds grant NNX16AI29G.

Software: GIZMO code (Hopkins 2015), ballic (Reinhardt \& Stadel 2017), VisIt.

\section{Appendix A Validation of the HLLC Riemann Solver}

We run the hydrostatic square test in Saitoh \& Makino (2013) and Hopkins (2015) using general EOS to test the HLLC Riemann solver at sharp boundaries. We initialize a two-dimensional fluid in a periodic box of Length $L=1$ (resolved by 128 particles) and uniform pressure $P=557.3$ (all in code units). We set ANEOS iron with $\rho=31$ within a central square of side-length $L=0.5$ surrounded by ideal gas with $\rho=15.5, \gamma=1.4$. The particles are evenly distributed but the iron particles' masses are twice of those of the gas particles. The sharp density contrast is well maintained at 44 sound crossing time (for the gas) in the MFM simulation and we observe no signs of deformation (Figure 7). Standard SPH cannot handle the sharp interface.

We then collide two uniform granite (Tillotson EOS) slabs $\left(15 \times 15 \times 8 R_{\oplus}\right)$ with opposite velocities. Both MFM and SPH in the GIZMO code can recover the Rankine-Hugoniot jump conditions (e.g., Melosh 1989), which shows the code's ability to correctly capture shocks (Reinhardt \& Stadel 2017). 


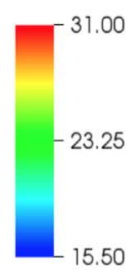

$$
t=44 t_{0}
$$

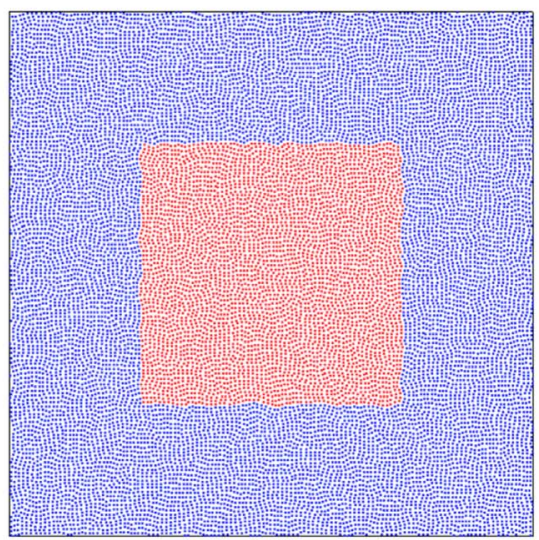

$t=4 t_{0}$

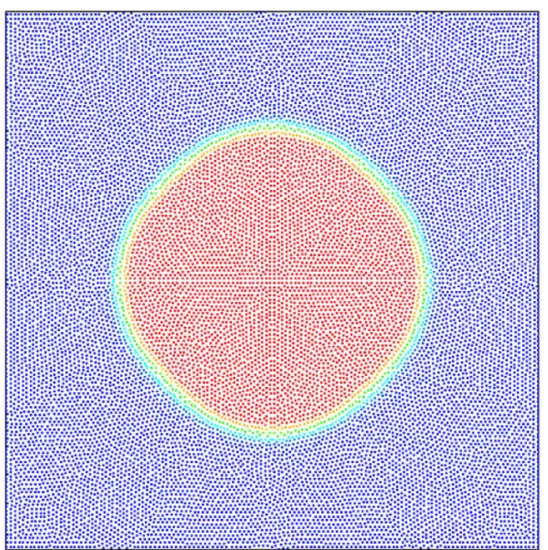

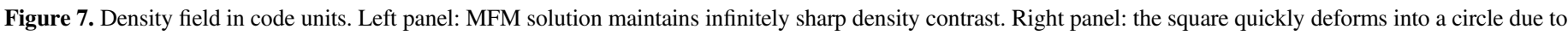
the artificial tension force in the standard SPH simulation.

\section{Appendix B}

\section{Entropy Changes Due to Phase Transitions}

Phase transitions can happen in impacts (Kraus et al. $2011,2015)$ so entropy conservation is not guaranteed in GIs even when there are no shocks. Pressure-release melting might ensue when the target is unloaded from highly compressed equilibrium state by the impactor (Asphaug et al. 2006). In the following tests we show that MFM can model phase transitions giving similar results to the CTH code in impact simulations. However, SPH cannot model phase transitions properly. We note that all the tests are run in the multimethod GIZMO code and all the comparisons are done with everything fixed except the factor we are discussing.

\section{B.1. Conservation Property}

We run two series of tests with the tabulated ANEOS EOS which has entropy information. The parameters for building the EOS table are set following Barr (2016). We take the iron core as an example to study the phase transitions.

First, our GIZMO code conserves entropy well both in SPH mode and MFM mode. Adiabatic expansion and pressurerelease melting is isentropic (Pierazzo et al. 1997). We run an adiabatic expansion test (Reinhardt \& Stadel 2017) by turning off the gravity of our $0.89 M_{\oplus}$ target model in Figure 2 to test the entropy conservation of MFM. The iron core has an initial entropy of $1200 \mathrm{~J} \mathrm{~kg}^{-1} \mathrm{~K}^{-1}$. At $50 \mathrm{hr}$, the target expands about 100 times in radius; the resolution decreases a lot. The entropy of the iron particles are well conserved with deviation smaller than 3\% for most particles (see Figure 8). At the core-mantle boundary, iron particles interact with the mantle leading to entropy nonconservation. MFM is able to conserve entropy well in the simulation timescale and the core-mantle boundary does not introduce systematic errors. SPH conserves entropy equally well in this test.

Second, our GIZMO code conserves the total energy well (internal energy plus kinetic energy and gravitational potential energy). We did an oscillation test on a hot $0.89 M_{\oplus}$ protoplanet $(\sim 500,000$ particles $)$ by adding $1 \mathrm{~km} \mathrm{~s}^{-1}$ radial velocities to particles beyond $0.7 R_{\oplus}$. The surface temperature of the protoplanet is $10,000 \mathrm{~K}$ and it has a fully molten core with an entropy of $1860 \mathrm{~J} \mathrm{~kg}^{-1} \mathrm{~K}^{-1}$ (Pierazzo et al. 1997). It

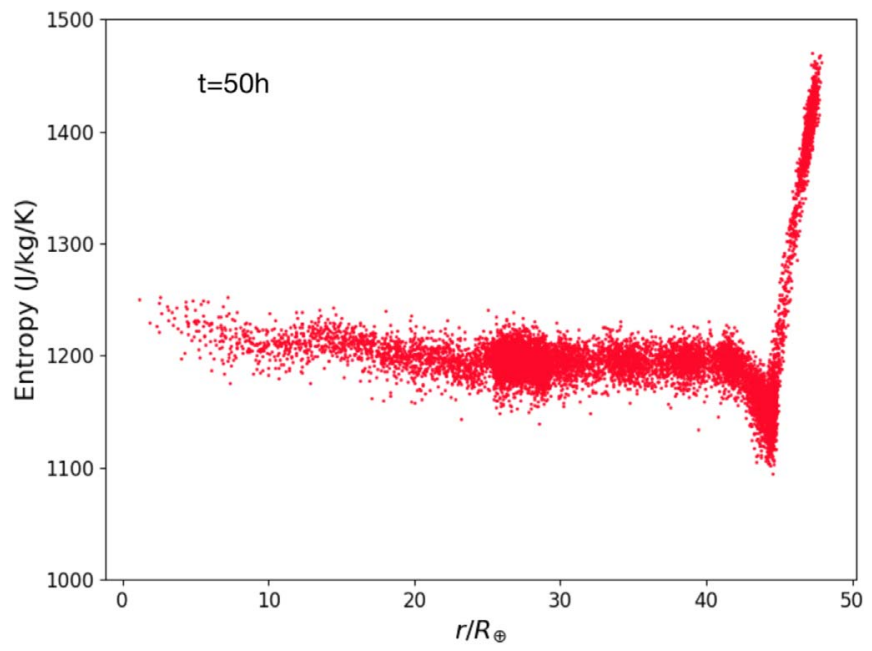

Figure 8. Entropy of the iron particles close to the equatorial plane $(5<z<5)$ in the MFM adiabatic expansion test.

oscillates radially and the errors of the total energy are within $2 \%$ in both MFM and SPH simulations. The core is fully molten and there are no phase transitions during the oscillations. The entropy of the core is well conserved shown in the phase diagram of Figure 9.

\section{B.2. Phase Transitions and Internal Energy Redistribution}

In reality the Earth does not have a fully molten core. In the moon formation impact simulations, the surface temperature of the proto-Earth is usually assumed to be $\sim 2000 \mathrm{~K}$ and the core is close to the melting curve (Alf et al. 1999; Anzellini et al. 2013; Barr 2016). When such a proto-Earth oscillates, pressurerelease melting starts at the outer core during the expansion; the outer core is more susceptible to melting than the inner core. Although pressure-release melting is isentropic here it is not allowed to expand freely. The total energy flux ignoring the source term of the gravitational energy is $\nabla\left(\rho u+\frac{1}{2} \rho v^{2}+P\right)$ (Hopkins 2015). The energy flux will soon reestablish quasi pressure equilibrium in the whole system. As a result, the melts near the CMB have higher internal energy than solid iron under 
the same pressure (see Figure 10 right panel). During the compression, the high internal energy melts result in net energy flux to the mantle leading to thermal energy extraction from the central core. We will show that this can be modeled with the ANEOS/M-ANEOS EOS in the following tests but not with the Tillotson EOS.

The Tillotson EOS lacks thermodynamically consistent treatment of mixtures between two phases and thus cannot model phase transitions (Brundage 2013). However, ANEOS can indeed model phase transitions (Melosh 2007). We run the

\section{Entropy $[\mathrm{kJ} / \mathrm{kg} / \mathrm{K}]$}
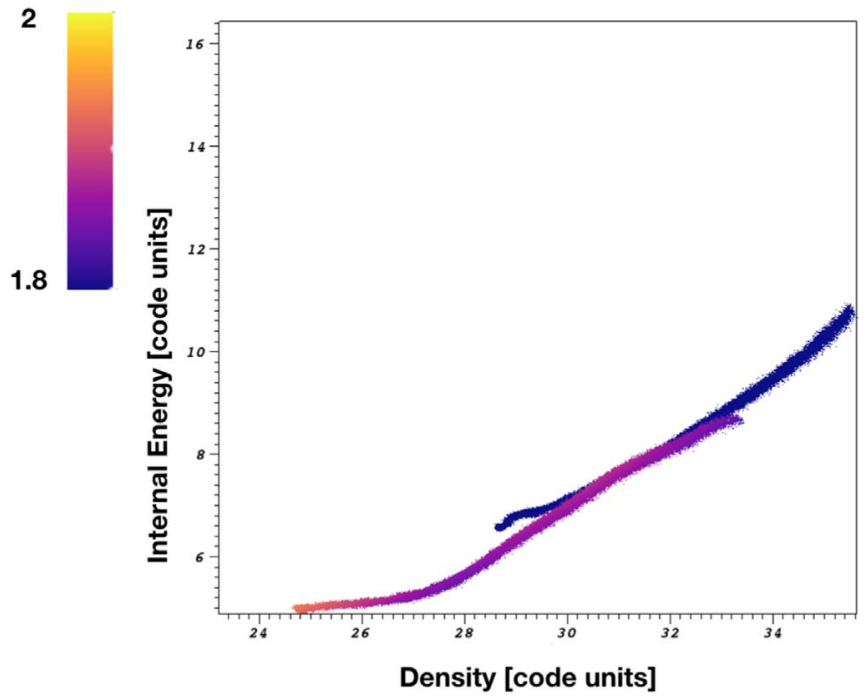

Figure 9. Phase diagram $(\rho-u)$ of the fully molten iron core in the MFM oscillation test. Particles lie on an isentrope with an entropy of $1860 \mathrm{~J} \mathrm{~kg}^{-1} \mathrm{~K}^{-1}$ initially (dark blue particles). The iron core oscillates along the isentrope but the entropy remains $\sim 1860 \mathrm{~J} \mathrm{~kg}^{-1} \mathrm{~K}^{-1}$ after $2.5 \mathrm{hr}$ (see the color-coded curve). oscillation test on the $0.89 M_{\oplus}$ target model (see Figure 2) whose core is close to the melting curve. We build another $0.89 M_{\oplus}$ target model using the Tillotson EOS. The mantle is granite instead of dunite. The iron core (blue particles) is slightly more compressed than that of the ANEOS/M-ANEOS model (red particles) but they lie on the same isentrope (1200 $\mathrm{J} \mathrm{kg}^{-1} \mathrm{~K}^{-1}$; see left panel of Figure 10).

In the Tillotson EOS simulation, the entropy conservation is good and the core oscillates along the isentrope. However, the outer core melts, according to Pierazzo et al. (1997) and Barr \& Citron (2011), in the ANEOS/M-ANEOS EOS simulation (see the right panel of Figure 10). This entropy change is not a numeric artifact because even the same test with a fully molten core conserves entropy precisely (see Figure 9). It is a sign of internal energy redistribution and phase transitions in the core.

\section{B.3. Comparison between Hydro-methods}

We run the oscillation test on a $0.89 M_{\oplus}$ protoplanet model (core entropy $1200 \mathrm{~J} \mathrm{~kg}^{-1} \mathrm{~K}^{-1}$ ) with $\mathrm{SPH}$ and ANEOS/MANEOS. The entropy of the core slightly increases (see Figure 11) due to numerical dissipation from the artificial viscosity (Springel 2005). The center of the core is strongly heated because strong artificial viscosity is wrongly triggered by the convergence flow during the compression (Cullen \& Dehnen 2010). We note that the central core's entropy increases by $300 \mathrm{~J} \mathrm{~kg}^{-1} \mathrm{~K}^{-1}$, which is much larger than the core entropy gain $\left(\sim 100 \mathrm{~J} \mathrm{~kg}^{-1} \mathrm{~K}^{-1}\right)$ due to the primary shock (at the first contact) in our simulations in Table 1. It shows no sign of phase transitions at the outer core because the pressure blips (see Figure 2) help to separate the core and mantle. This numerical separation impairs energy flux and keeps the core adiabatic to some extent.

The CTH code is well tested with the ANEOS/M-ANEOS EOS (Crawford et al. 2006). Unfortunately, we were not able to run the oscillation test with the CTH code. We checked the entropy profile of the post-impact target as a function of the
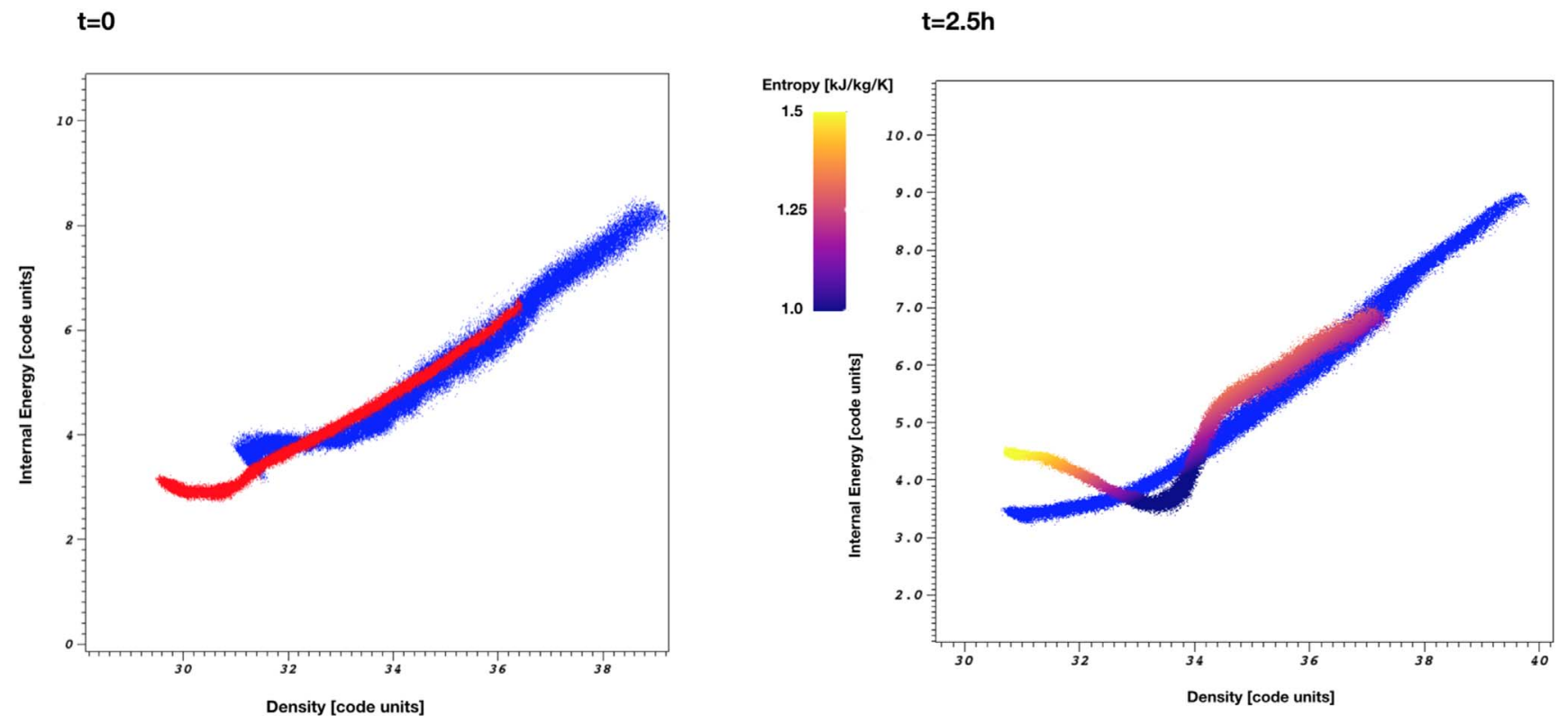

Figure 10. Phase diagram $(\rho-u)$ of the iron core in the MFM oscillation test with the Tillotson EOS (blue particles) and ANEOS/M-ANEOS EOS (red or color-coded by the entropy). Particles lies on an isentrope with an entropy of $1200 \mathrm{~J} \mathrm{~kg}^{-1} \mathrm{~K}^{-1}$ initially, shown in the left panel. The iron core oscillates along the isentrope when we use the Tillotson EOS. In the simulation with the ANEOS/M-ANEOS EOS, the outer core melts due to pressure release and the internal energy of the core redistributes. 
Entropy $[\mathbf{k J} / \mathbf{k g} / \mathbf{K}]$

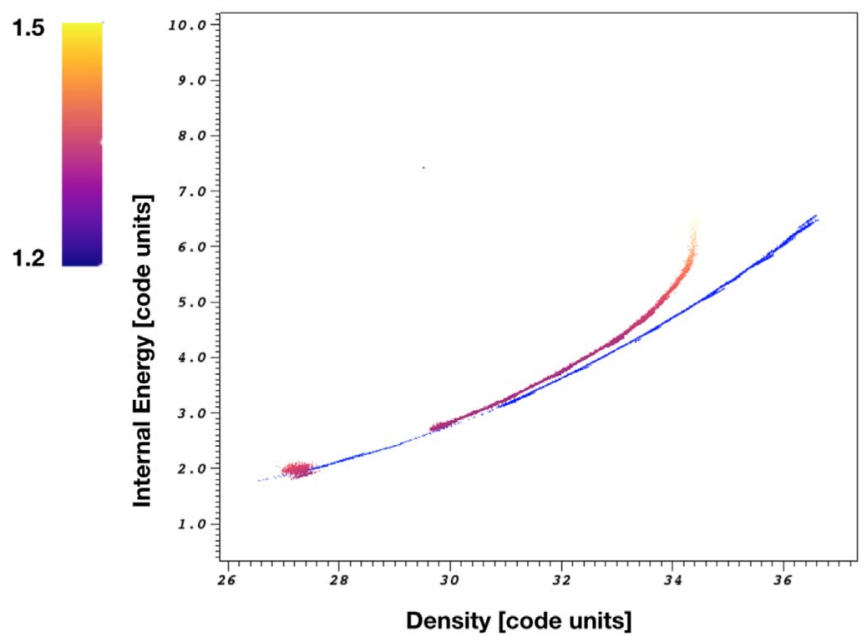

Figure 11. Phase diagram $(\rho-u)$ of the iron core in the SPH oscillation test Particles lies on an isentrope with an entropy of $1200 \mathrm{~J} \mathrm{~kg}^{-1} \mathrm{~K}^{-1}$ initially (blue particles). The iron core oscillates along the isentrope but the entropy increases $\sim 300 \mathrm{~J} \mathrm{~kg}^{-1} \mathrm{~K}^{-1}$ in the central core after $2.5 \mathrm{hr}$ (see the color-coded curve).

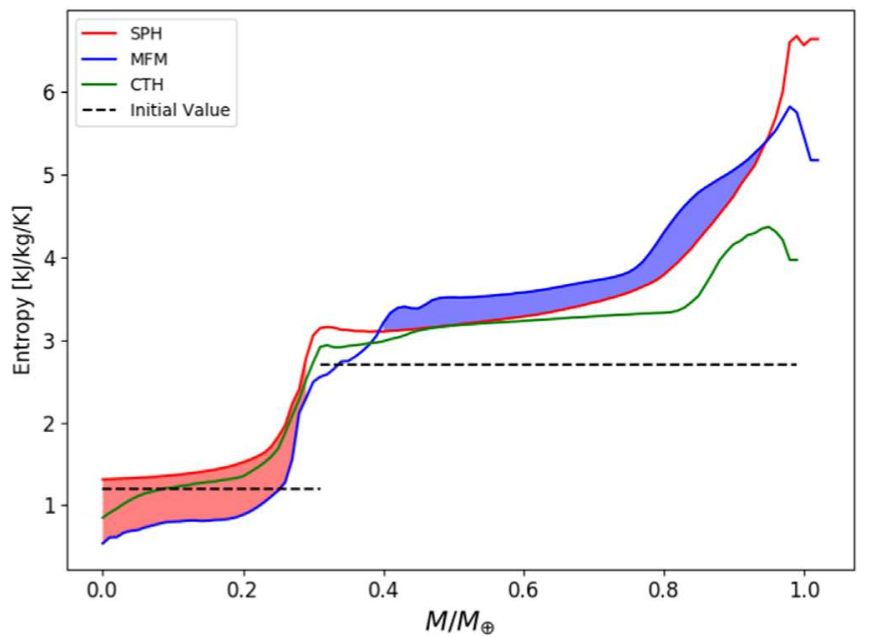

Figure 12. Entropy profile as a function of normalized enclosed mass in the CTH and GIZMO simulation $(t=40 \mathrm{hr})$ of run119 with the initial entropy indicated by the black dashed lines. The central core's entropy decreases in the CTH and GIZMO MFM simulation, which is absent in the GIZMO SPH simulation. The difference in entropy around the core-mantle boundary is likely caused by the different treatment of material interfaces.

normalized enclosed mass in the benchmark run119 (Barr 2016) by CTH and GIZMO using about $2 \mathrm{M}$ cells/particles.

First, the entropy structure of the mantle (in Figure 12) agrees well with Nakajima \& Stevenson (2015) when we run GIZMO in SPH mode validating again our EOS implementation. In Figure 12, parts of the post-impact target's core have even lower entropy than their initial values $\left(1200 \mathrm{~J} \mathrm{~kg}^{-1} \mathrm{~K}^{-1}\right.$, indicated by the black dashed lines) in both GIZMO MFM and CTH. It is known well that the shocks deposit thermal energy and increase the entropy. The entropy decrease can be explained by phase transitions in the outer core and the following internal energy redistribution (lost to the mantle) as discussed above. The red and blue shaded regions are of almost equal area and they measure the extra thermal energy transport from the core to the mantle in the MFM run compared to the SPH run.
The two codes are very different by nature because CTH is an AMR Eulerian code and GIZMO is a Lagrangian code. The treatment of material interface is also different. In GIZMO (both MFM and SPH mode) every computational element is either iron or rock but CTH allows cells with both rock and iron contributions. Given all the differences above, the qualitative agreement in the thermal structure of the post-impact target (see Figure 12) is satisfactory.

\section{ORCID iDs}

Hongping Deng (ib https://orcid.org/0000-0001-6858-1006

Amy C. Barr (ํ) https://orcid.org/0000-0002-1278-0003

\section{References}

Agertz, O., Moore, B., Stadel, J., et al. 2007, MNRAS, 380, 963

Alf, D., Gillan, M., \& Price, G. 1999, Natur, 401, 462

Anzellini, S., Dewaele, A., Mezouar, M., Loubeyre, P., \& Morard, G. 2013 , Sci, 340, 464

Asphaug, E. 2014, AREPS, 42, 551

Asphaug, E., Agnor, C. B., \& Williams, Q. 2006, Natur, 439, 155

Balbus, S. A., \& Hawley, J. F. 1991, ApJ, 376, 214

Balsara, D. S. 1995, JCoPh, 121, 357

Barr, A. C. 2016, JGRE, 121, 1573

Barr, A. C., \& Citron, R. I. 2011, Icar, 211, 913

Bauer, A., \& Springel, V. 2012, MNRAS, 423, 2558

Beck, A. M., Murante, G., Arth, A., et al. 2016, MNRAS, 455, 2110

Benz, W., Slattery, W., \& Cameron, A. 1986, Icar, 66, 515

Brundage, A. L. 2013, Procedia Engineering, 58, 461

Cameron, A. G., \& Ward, W. R. 1976, LPSC, 7, 120

Canup, R. M. 2012, Sci, 338, 1052

Canup, R. M., \& Asphaug, E. 2001, Natur, 412, 708

Canup, R. M., Barr, A. C., \& Crawford, D. A. 2013, Icar, 222, 200

Chambers, J. 2001, Icar, 152, 205

Crawford, D., Taylor, P., Bell, R., \& Hertel, E. 2006, Russ. J. Phys. Chem., 25,85

Ćuk, M., \& Stewart, S. T. 2012, Sci, 338, 1047

Cullen, L., \& Dehnen, W. 2010, MNRAS, 408, 669

Dauphas, N. 2017, Natur, 541, 521

Deng, H., Mayer, L., \& Meru, F. 2017, ApJ, 847, 43

Emsenhuber, A., Jutzi, M., \& Benz, W. 2017, Icar, 301, 247

Fryxell, B., Olson, K., Ricker, P., et al. 2000, ApJS, 131, 273

Garcia-Senz, D., Cabezon, R. M., \& Escartin, J. A. 2012, A\&A, 538, A9

Gingold, R. A., \& Monaghan, J. J. 1977, MNRAS, 181, 375

Hopkins, P. F. 2013, MNRAS, 428, 2840

Hopkins, P. F. 2015, MNRAS, 450, 53

Hopkins, P. F. 2017, arXiv:1712.01294

Hosono, N., Saitoh, T. R., \& Makino, J. 2013, PASJ, 65, 108

Hosono, N., Saitoh, T. R., Makino, J., Genda, H., \& Ida, S. 2016, Icar, 271, 131

Hu, X., Adams, N., \& Iaccarino, G. 2009, JCoPh, 228, 6572

Kaib, N. A., \& Cowan, N. B. 2015, Icar, 252, 161

Kraus, R. G., Root, S., Lemke, R. W., et al. 2015, NatGe, 8, 269

Kraus, R. G., Senft, L. E., \& Stewart, S. T. 2011, Icar, 214, 724

Leinhardt, Z. M., \& Stewart, S. T. 2012, ApJ, 745, 79

Liu, S.-F., Hori, Y., Lin, D., \& Asphaug, E. 2015, ApJ, 812, 164

Lucy, L. B. 1977, AJ, 82, 1013

Mastrobuono-Battisti, A., Perets, H. B., \& Raymond, S. N. 2015, Natur, 520,212

McGlaun, J. M., Thompson, S., \& Elrick, M. 1990, IJIE, 10, 351

Melosh, H. 2007, M\&PS, 42, 2079

Melosh, H. J. 1989, Research supported by NASA, Oxford Monographs on Geology and Geophysics, Vol. 253 (New York: Oxford Univ. Press), 11

Nakajima, M., \& Stevenson, D. J. 2015, E\&PSL, 427, 286

Ott, F., \& Schnetter, E. 2003, arXiv:physics/0303112

Pierazzo, E., Vickery, A., \& Melosh, H. 1997, Icar, 127, 408

Price, D. J. 2008, JCoPh, 227, 10040

Read, J., \& Hayfield, T. 2012, MNRAS, 422, 3037

Reinhardt, C., \& Stadel, J. 2017, MNRAS, 467, 4252

Reufer, A., Meier, M. M., Benz, W., \& Wieler, R. 2012, Icar, 221, 296

Rosswog, S. 2015, MNRAS, 448, 3628

Saitoh, T. R., \& Makino, J. 2013, ApJ, 768, 44 
Saitoh, T. R., \& Makino, J. 2016, ApJ, 823, 144

Solenthaler, B., \& Pajarola, R. 2008, in Eurographics/SIGGRAPH Symposium on Computer Animation, ed. M. Gross \& D. James (Geneve: The Eurographics Association)

Springel, V. 2005, MNRAS, 364, 1105

Thompson, S., \& Lauson, H. 1974, Improvements in the Chart D radiationhydrodynamic CODE III: Revised analytic equations of state, Tech. rep. No. SC-RR-71-0714 (Albuquerque, NM: Sandia Labs)
Tillotson, J. H. 1962, Metallic equations of state for hypervelocity impact, Tech. rep. No. GA-3216 (San Diego, CA: General Dynamics, Atomic Div.) Toro, E. F., Spruce, M., \& Speares, W. 1994, ShWav, 4, 25

Wadsley, J. W., Keller, B. W., \& Quinn, T. R. 2017, MNRAS, 471, 2357

Wiechert, U., Halliday, A., Lee, D.-C., et al. 2001, Sci, 294, 345

Woolfson, M. 2007, MNRAS, 376, 1173

Zhang, J., Dauphas, N., Davis, A. M., Leya, I., \& Fedkin, A. 2012, NatGe, 5,251 